\title{
PARAMETERS OF TACHISTOSCOPIC STEREOPSIS ${ }^{1}$
}

\author{
William R. Uttal, Jldy Fitzgerald and Thelma E. Eskin ${ }^{2}$ \\ The University of Michigan. Ann Arbor, Michigan 48104, U.S.A.
}

(Received 15 July 1974; in revised form 3 October 1974)

\begin{abstract}
This study examines the effects of disparity, dot numerosity, exposure duration. and the allowed processing time on briefly exposed Julesz-type random dot stereogram perception. Dot numerosity produces only minimal effects for the lower values used; reductions in exposure duration systematically reduce the performance level; and the changes in performance are found to be symmetrical for either convergent or divergent disparity and to continuously improve with disparity varying from approx $30^{\prime \prime}$ to over $5^{\prime}$ of visual angle. The findings from this experiment indicate that there is only minimal summatory interaction among stereoscopic mechanisms for adjacent areas and confirm earlier estimates of the time required (approx $50 \mathrm{msec}$ ) for processing stereoscopic stimuli.
\end{abstract}

\section{INTRODUCTION}

The random dot stereoscopic technique, as developed to its present high level by Bela Julesz and summarized in his important book (Julesz, 1971), provides an extraordinary opportunity to study a pure form of depth perception that is dependent only upon disparity cues. However, various practical aspects of the technique have hampered development of certain important lines of research. particularly those involving brief exposure and real-time control of several key independent variables. Typically, although there have been a few exceptions (e.g. Papert, 1964 and Marlowe, 1969), the random dot stereograms used by Julesz followers are generated on the face of an oscilloscope, photographed, and then re-presented to the $S$ in a conventional optical stereoscope. This photographic procedure is fairly cumbersome, rather expensive, and ponderously time-consuming. Thus, parametric investigations that are necessary to understand such dimensions as dot numerosity, exposure time, and sequential interaction in stereoscopic viewing have been carried out infrequently, in spite of the fact that there has long been an awareness of these problems in the study of stereopsis (e.g. Dove, 1841).

Several laboratories have recently developed procedures in which small laboratory computers control oscilloscope displays directly. The computer operates rapidly enough to regenerate patterns and to produce dynamic effects that increase our understanding of the richness of the stereoscopic process. Ross and Hogben (1974), for example, have used such a system to demonstrate that the persistence of the stereoscopic trace is sufficiently long to allow the perception of depth without degradation when the intervals between the stimuli presented to each eye are separated by $36-72 \mathrm{msec}$. Ross (1974) has also shown that time discrepancies, in

\footnotetext{
The research reported in this paper was supported by research grants from the National Science Foundation (GB25431), the National Institute of Mental Health (1 ROI MH24016-01), and the Rackham Graduate School of The University of Michigan, and by an NIMH Research Scientist Award (5 KO5 MH29941-03) to the first author.

${ }^{2}$ We would like to express our appreciation to Gregory Zick who assisted in the construction of the three-dimensional analog computer circuitry for the hybrid computer system.
}

some instances, can be substituted for spatial disparities to produce stereoscopic depth in a manner not explicable in terms of the actual spatial disparities produced by stimulus intensity differences in situations like that underlying the Pulfrich phenomenon.

The present study reports a similar approach to another set of problems; i.e. the effects of duration of exposure and dot numerosity on stereopsis. Experiments were carried out to determine the effects of these parameters both with and without a backward masking, or "blanking", field to determine stereoscopic perception time. A follow-up study investigated the stereoscopic effectiveness of a severely reduced display which consisted of just two briefly exposed dots. In all experiments, the procedure used was efficient enough to explore the full effective range of retinal dispartiy.

\section{METHOD}

Subjects

The Ss for each experiment were four undergraduate students at The University of Michigan who were paid stipends for a daily participation period of one hour. Because the time period for the experiments was long, different students participated in each of the three experiments; thus crosscomparisons among absolute levels are not meaningful. In spite of the fact that $S$ s were pretested with anaglyphs from Julesz (1971) book, a wide range of individual differences in stereoscopic viewing competence was opserved among Ss in the context of this experimental procedure. Some Ss proved to be stereo-deficient and their data were removed from the final analyses. In no instance were the data of fewer than three Ss used to plot any of the figures.

\section{Apparatus}

The experiments were controlled by a hybrid computer system that used both digital and analog methods to make the calculations required for the generation of stereoscopic displays. The digital computer was a Digital Equipment Corporation PDP-8/E and the analog operational modules were manufactured by Optical Electronics, Inc.

The calculation of the coordinates of the dots composing the stereoscopic display was carried out digitally on the PDP-8/E. The programmer defined the characteristics of the stimuli in general (e.g. specifying the extent of a plane and placing it at an assigned depth in the $S$ s three-dimensional space). The locations of the dots within the specified area were then individually determined by random number 705 


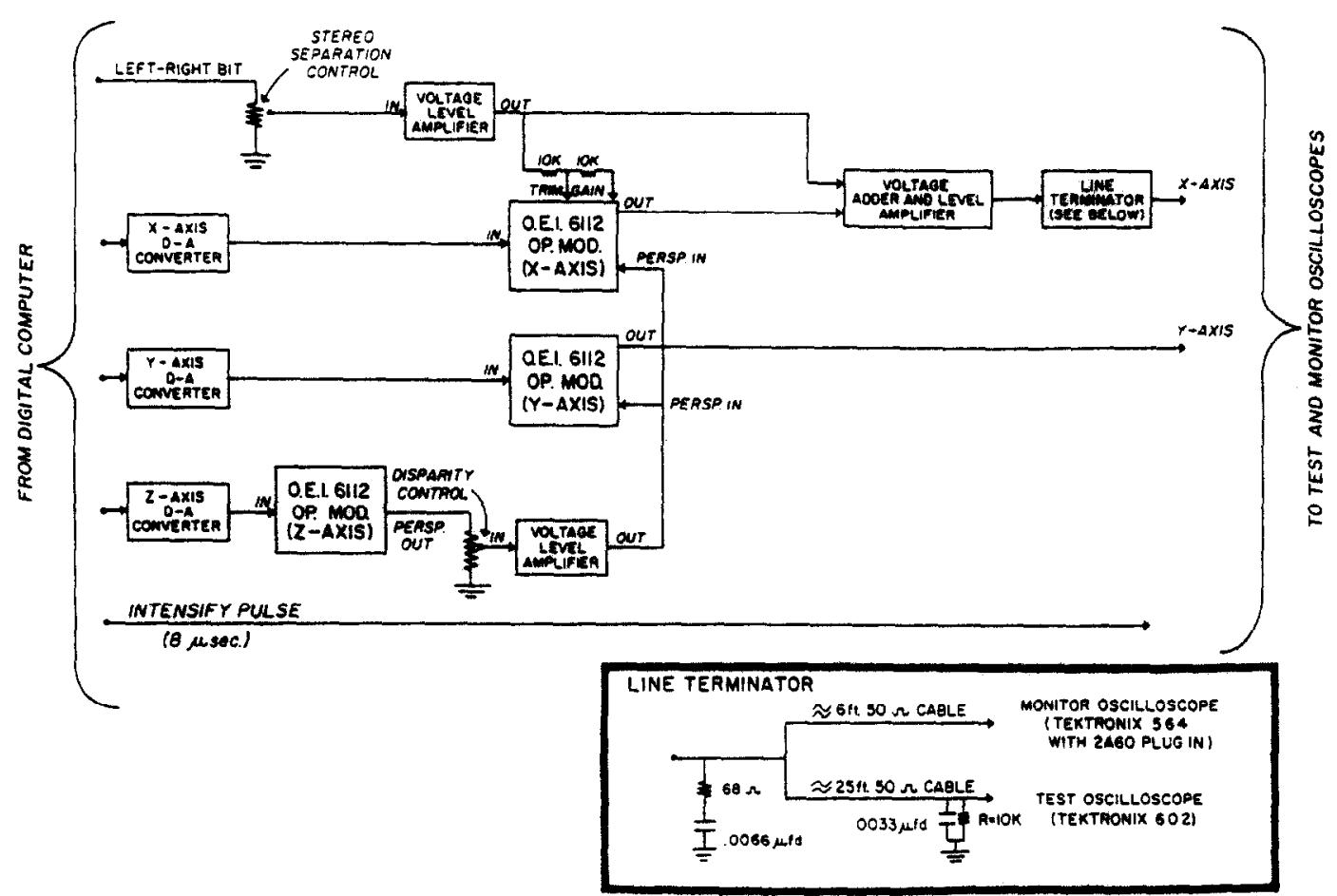

Fig. 1. Block diagram of the arrangement of the analog computing elements in the hybrid computer system. Encoded information is introduced into this subsystem from the digital computer through three digital-to-analog converters. The analog subsystem then processes this information and a single "left-right" bit (see text for details) to produce appropriately coded $x$ and $y$ axis deflection signals for plotting twodimensional stimuli for each eye on the face of a conventional oscilloscope. Upon proper convergence, a strong perception of depth is produced. The line-terminating resistor-capacitor network is required :0 reduce parasitic oscillations introduced as a result of impedance mismatches between the cables and the analog computing elements.

composing each stimulus was built up in the digital computer's memory. This table consisted of three coordinates for each dot: $x$, the horizontal position: $y$, the vertical position; and $z$, the depth in the cubicle volume defined by the $S \mathrm{~s}$ stereoscopic perception. These digital coordinate values were converted to analog voltages by three 10-bit digital-toanalog converters. The outputs of these converters were then fed into the analog operational modules.

The final calculations and the plotting of the dots were controlled by the analog operational modules. These circuit devices formed a subsystem that plotted two actual dots for each stereoscopic dot, one in the left eye's field of view and one in the right eye's field of view, with a disparity appropriate to the $=$ coordinate defined in the computer program. A block diagram of this analog portion of the hybrid computer system is shown in Fig. 1 .

The inputs to the analog operational modules are of four kinds: the $x, y$, and $z$ coordinate information and a one-bit signal indicating that the display should plot a dot either in the right or left eye's field of view. The $z$ axis information and the left-right bit together define exactly where the pair of dots representing a stereoscopic dot with specific $x, y$, and $x$ coordinates will be plotted on the face of a Tektronics 602 oscilloscope. The $z$ axis signal is an additional voltage input to the $x$ and $y$ axis operational modules that alters the digitally computed values of these two coordinates to produce the correct disparity that must be associated with the specified z axis coordinate. The "left-right" bit laterally displaces the dot to either side of the stereoscope, while maintaining the correct disparities defined by the $x, y$, and $z$ coordinates for each eye's view. The amplifiers indicated in Fig. 1 were used solely to adjust the voltage levels and polarities to compatible levels for the various parts of the system.

The $S$ viewed the oscilloscope screen, which was divided by an opaque septum so that each eye's field of view was isolated, through two converging prisms adjusted for comfor- table dichoptic fusion at a distance of $33 \mathrm{~cm}$. Since the plotted disparity is a function of the arbitrary setting of an uncalibrated control associated with the analog circuitry, there was no a priori relationship between the computed $z$-axis coordinate value and the retinal disparity. Disparities were, therefore, directly calibrated by plotting the test pattern shown in Fig. 2 on the face of the oscilloscope. The actual disparities employed are indicated for each experiment below. The binocular viewing field was $14^{\prime}$ in width, and each eye viewed a $5.6^{\circ} \times 5.6^{\circ}$ field.

The calculations of the disparity and left-right position of the two images of each dot were carried out by the analog circuitry virtually instantaneously; the analog circuitry had a band pass of $500 \mathrm{kHz}$ and a slewing rate of $100 \mathrm{~V} / \mu \mathrm{sec}$. Thus, a much smaller computation load is placed upon the digital computer at the critical time of plotting and the display is able to operate almost as fast as a two-dimensional dot plotter; i.e. at speeds of approx 12 plotted dots per msec. The oscilloscope phosphor itself is also very fast-decaying to 0.1 per cent of its initial brightness in less than $50 \mu \mathrm{sec}$. Because of these features and the persistence of the visual image, this system is able to plot stereoscopic stimuli that appear to be composed of simultaneously plotted dot patterns. However, for the shortest exposures (e.g. $5 \mathrm{msec}$ ) and largest number of dots (e.g. 50), the exposure durations are nominal and represent the duration of the dot stream and not the period over which each dot was repetitively plotted.

\section{Procedure}

Stimulus conditions and the order of the target and nontarget condition in each trial were randomly selected by a computer algorithm from among a predetermined set of stimuli. Generally, one other parameter, the main independent variable for each of the experiments, was varied daily and specified when the initial instructions were loaded into the computer. Ss signed themselves on and off, and the pro- 


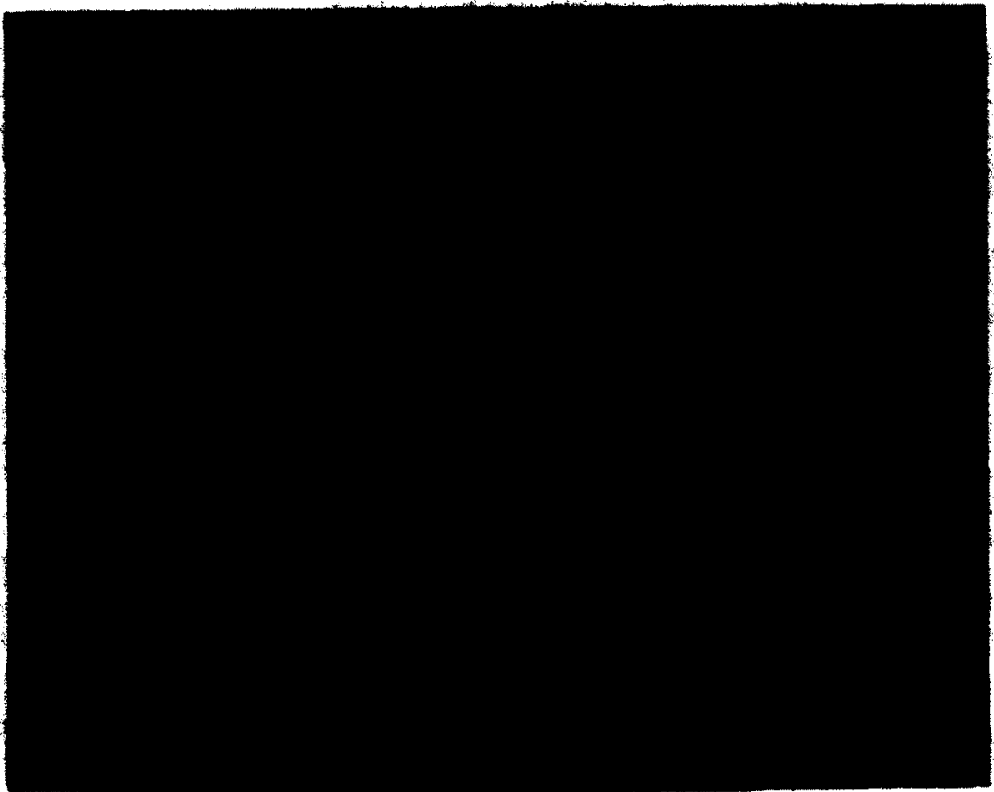

Fig. 2. Photograph of a stereoscopic test pattern generated for the calibration of the disparity variable used throughout the study. Upon proper fusion, this figure appears as a dotted outline cube with a dotted line that is closer to the observer at the top and further away at the bottom.

tocol of each day's session was managed completely by the computer. A daily data analysis obtained the number of trials, the number of correct responses, and the percentage of correct responses for each $S$ as well as the summary of this information for all Ss. Approximately 800 trials were executed for each $S$ hour. The number of trials used in the estimate of each point in the figures varied from 100 to 3200 . depending upon the degree of pooling for each graph.

The $S s$ task in each of the experiments was to choose which of two sequential stimuli. each of which consisted of dichoptically presented disparate patterns of dots, contained a stereoscopic target condition in a two-alternative, forced-choice paradigm. For example, the $S$ was required in some cases to determine which of two stimuli contained two planes at different depths as opposed to one that had the same number of dots but all located at the same depth, and in another case to choose which of two sequentially presented pairs of dots exhibited a depth difference. The $S$ responded by depressing one of two hand-held push buttons. A plus or a minus sign was then displayed on the oscilloscope screen to serve as feedback, indicating whether or not the response was correct. These feedback symbols, which preceded the first stimulus pattern by $500 \mathrm{msec}$, also served as fixation guides and provided additional references for correct convergence in an otherwise dark, sound-shielded experimental cubicle. The $S$ 's head was constrained to a fixed position by a forehead pressure switch that inactivated the response switches if the $S s$ head was improperly placed. The rims of the converging lenses also assured correct lateral head position. The dependent variable in all cases was the percentage of the total number of presented stimuli to which the $S$ responded correctly. In all instances, 50 per cent correct must be considered chance performance level because of the two-alternative, forced-choice procedure.

\section{Experiment 1}

The first experiment was designed to determine the effect of viewing duration. dot numerosity, and disparity on stereo. scopic depth perception. Two stereoscopic planes were plotted perpendicularly to the $S_{s}$ s line of sight. One, a refer. ence plane, extended over the full range of the $5.6^{\circ} \times 5.6^{\circ}$ viewing field for each eye. The $S$ converged on dots at the depth of this plane whose $z$-axis coordinate thus defined zero disparity. The other. a test plane, extended over a smaller field subtending $2.8^{\circ} \times 2.8^{\circ}$. The test plane contained one-fourth the number of dots in the reference plane. The $z$ axis of the test plane could be specified to locate it either in front of or in back of the reference plane by crossing (converging) or uncrossing (diverging) the disparity. The viewing time and the number of dots in both planes were also independent variables.

In both this experiment and Experiment 2, the two sequential stimuli had the same dot density and distribution in the $x, y$ plane. An additional group of random dots, equivalent in number and extent to those in the test plane, was added to the nontarget stimulus, but located at the zero-disparity z-axis depth to negate any secondary cues of density or pattern in the experimental task. The two sequential stimuli were separated by an interval of 1 sec.

This experiment was run in three parts. The first part explored the effect of disparity and dot numerosity at an exposure duration of $500 \mathrm{msec}$. Reference plane dot numer. osities of $4,10.30,50,100,200,300$ and 377 were used in this part of the experiment. In each case, the number of dots in the test plane was equal to one-fourth the number in the reference plane. Eleven steps of disparity were used varying from $5.60^{\circ}$ (crossed) to $0-5.60^{\circ}$ (uncrossed) in $1.12^{\prime}$ steps. No vergence aid other than the plus or minus feedback signal and the stimuli themselves was used in this part of the experiment.

In the second part of the experiment, stimuli were exposed for $5 \mathrm{msec}$. The same disparities were used as in the first part but only reference plane dot numerosities of 50 . 30 , 10. and 4 could be tested because of the shortened dis* play time. A pair of vergence adjusting fixation dots was displayed during the intervals between the feedback signal and the first stimulus and between the first and second stimuli in order to eliminate the possibility that vergence drifts would interfere with the perception of these brief stimuii.

The third part of Experiment 1 provided the link between the first two parts by determining the specific effect of expo. sure duration on stereoscopic depth. This part of the experiment always used stimuli that contained 100 dots in the reference plane, 25 in the test plane, and the same disparity schedule used in the first part of the experiment. but scanned the exposure duration dimension by using the following values: $500,400,200,100,50,40$ and $20 \mathrm{msec}$. No vergence adjusting point was used here. 


\section{Experiment 2}

The second experiment was identical in procedure to the first, with the exception that as in Julesz" (1964) experiment, a masking burst of 250 random dots followed the display of each of the two stimuli at a variable interval. In using this procedure, we hoped both to substantiate the estimate of processing time required for stereopsis obtained by Julesz and to determine the function over a more complete range of stimulus-mask intervals. A single numerosity condition was used for this experiment: stimuli consisted of 100 dots in the test plane presented against a reference plane consisting of 250 dots. A masking burst of 250 dots plotted at intervals of $1.10,20,30,40,50,100,150$ and $200 \mathrm{msec}$ followed the test and reference planes. Only one interval between the stimulus and blanking mask was used each day, but disparities were scanned daily by randomly selecting among values that also varied between $5.60^{\circ}$ (crossed) and $5.60^{\circ}$ (uncrossed) in $1 \cdot 12^{\prime}$ steps. Two values of exposure duration were also examined-40 and $20 \mathrm{msec}-$ to determine if viewing time was an important factor in this type of masked stereoscopic viewing.

\section{Experiment 3}

The third experiment reduced all stereoscopic parameters-numerosity, exposure duration. and disparity conditions-to minimum levels. Only two dots were used in each stimulus; the $S$ was required to specify which of two sequentially presented pairs of dots differed in depth. The purpose of this experiment was to determine the variation in stereopsis as a function of disparity under these severely reduced conditions. A dichoptic pattern composed of the four corners of the viewing field was used so that vergence would be stable and the images fused when the pair of dots was flashed. The dot stimuli were intensified only once and the exposure duration was. therefore, equivalent to the decay time of the phosphor $(50 \mu \mathrm{sec})$. The major independent variable in this experiment was disparity ranging from $5.60^{\prime}$ (crossed) to $5.60^{\prime}$ (uncrossed) in approx $0.51^{\prime}$ steps.

\section{RESLLTS}

\section{Experiment 1}

Figures 3,4 , and 5 display the results of the three parts of Experiment 1. Figure 3 shows the effects of

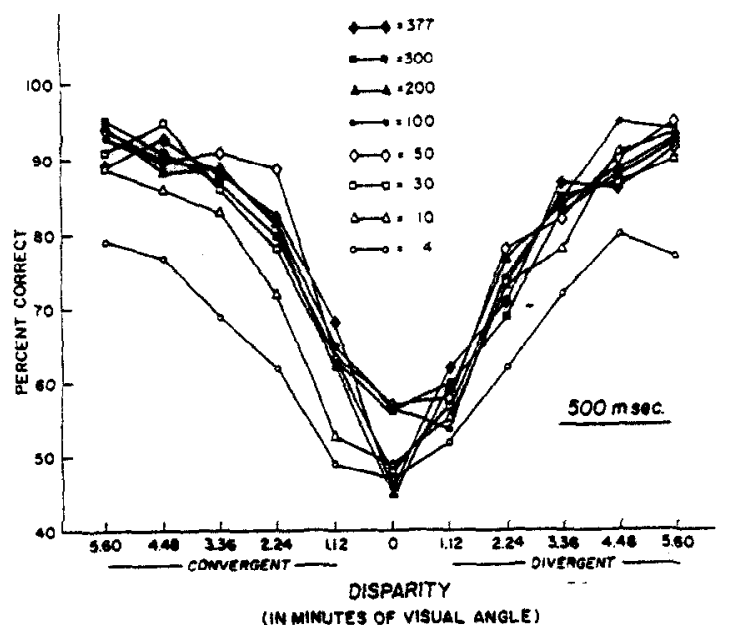

Fig. 3. The results of the first part of Experiment 1. At the prolonged exposure duration used ( $500 \mathrm{msec}$ ) there is little effect of dot numerosity except at the lowest value; however. the effect of disparity is strong. Ss continuously and symmetrically improve their performance with increases in both convergent and divergent disparity. The family of curves is parametric with the number of dots in the reference plane. This part of the experiment did not use a convergence aid.

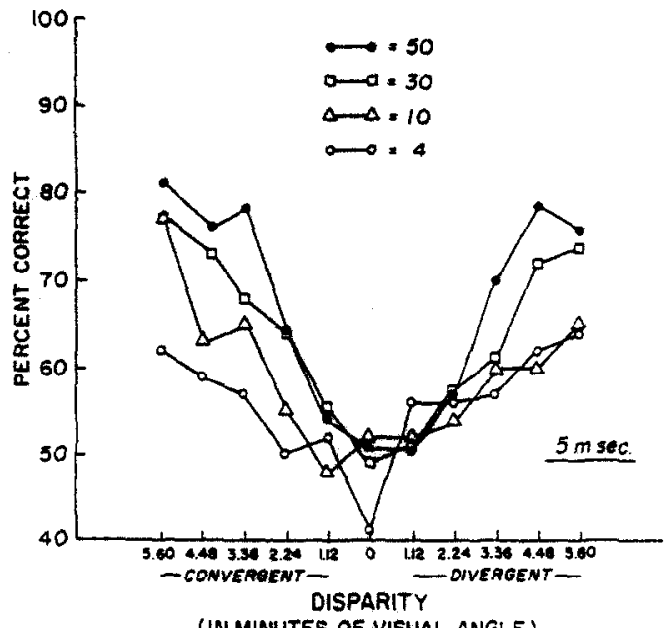

Fig. 4. The results of the second part of Experiment I. At this brief exposure duration $(5 \mathrm{msec})$ the effect of dot numerosity is greater than in Fig. 3, but the same continuing and symmetrical increase in sensitivity with increases in disparity is observed. This part of the experiment used a convergence aid. The family of curves is parametric with the number of dots in the reference plane.

both disparity and dot numerosity on the stereoscopic perception of two planes exposed for $500 \mathrm{msec}$. As the more or less symmetrical limbs of the curve indicate for crossed and uncrossed disparities, respectively, there is a gradual decline in the ability of $S$ to discriminate between a reference and a test plane as the disparity decreases from a maximally crossed disparity of $5.60^{\circ}$ to zero disparity, and then a gradual increase in the discriminability as the disparity once again increases to the maximum uncrossed value of $5 \cdot 60^{\circ}$. Although it is not possible to generalize for all parametric curves, it is clear that, for at least some cases, there is a measurable difference between zero disparity and the next smallest value $\left(0.51^{\prime}\right)$. Thus, in some instances, when employing this task and instrumentation, stereopsis appears to be present for disparities of less than $30^{\circ}$ of arc. While this value may be considered to be a possible threshold for the present experimental task. the overall statistics of the continuous function generated are of greater interest.

The effect of dot numerosity, on the other hand. is small. With the exception of the two lowest values (4 and 10 dots in the reference plane and 1 and 3 dots in the test plane), the curves seem to lie one on top of another. The 10-dot condition has a slightly lower value than the others only for the crossed disparity condition, while the 4-dot condition averages about 15 per cent lower than the cluster of other curves for both crossed and uncrossed conditions. The best performance level obtained with maximum disparity and dot numerosity is 95 per cent for this relatively long exposure duration of 500 msec.

Figure 4 presents the results of the second part of Experiment 1 in which the exposure duration was only 5 msec. In this case, the best performance level was about 80 per cent--indicating a considerable deficit solely as a function of the exposure duration. This occurred in spite of the fact that a vergence-aiding dot was used in this brief exposure condition. Furthermore, there appears to be a somewhat larger differen- 


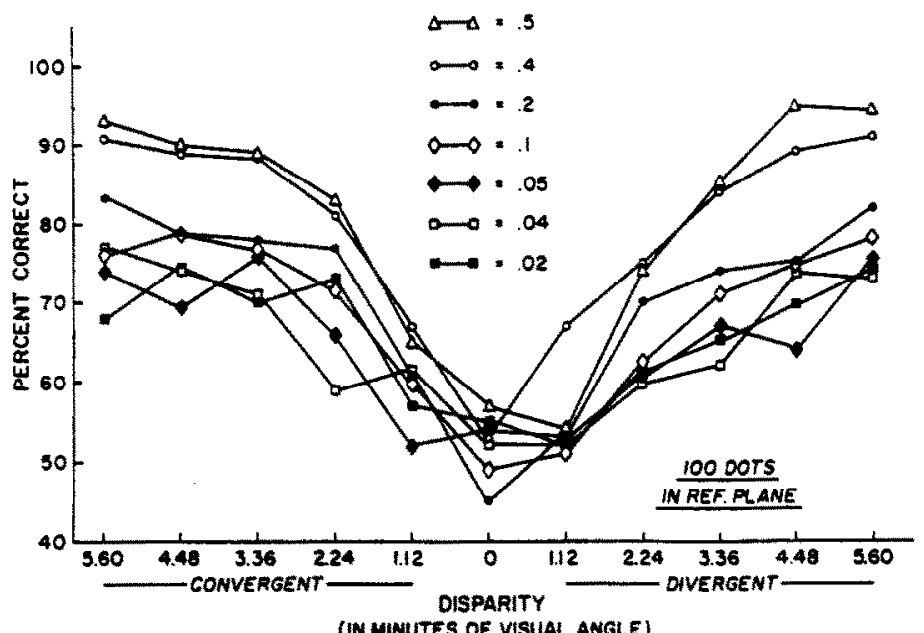

Fig. 5. The results of the third part of Experiment 1 . The effect of reducing the viewing duration is seen to progressively reduce the performance level. Nevertheless, the same pattern of symmetrical and continuous increase in performance as either convergent or divergent disparity is increased is seen at all durations. The family of curves is parametric with the viewing duration measured in seconds.

tial effect of dot numerosity for this shorter exposure duration. The typical difference between the corresponding scores for the 50-dot condition and for the 4 dot condition is about 20 per cent in Part II, compared to 10 per cent for the longer durations of Part I.

The results of the third part of Experiment 1 are shown in Fig. 5 . In this case, the disparity curves have been presented parametrically as a function of exposure duration. Clearly, the effect of exposure duration is to reduce the stereoscopic discriminability of the two planes. The absolute values of the lower curves (100 dots at 40 and $20 \mathrm{msec}$ ) in this figure are lower than those of the curve for 50 dots at 5 msec in Fig. 4, indi-

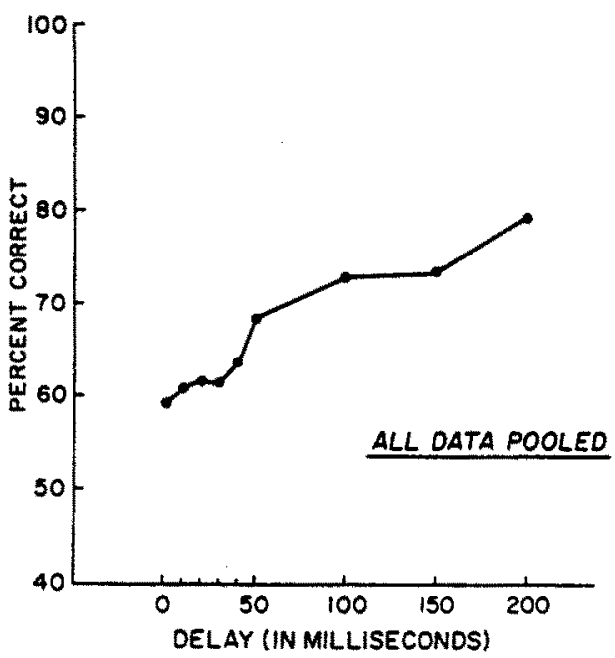

Fig. 6. The results of Experiment 2 in which a blanking field was used to interfere with the processing of the stereoscopic percept. Data from all parts of the experiment have been pooled in plotting this figure. There is a discontinuity at about 50 msec but no great increase in performance at shorter or longer stimulus-mask intervals. This discontinuity suggests that there is, as suggested by Julesz (1964) a 50 msec processing period required for stereoscopic perception. cating that the convergence adjustment point substantially helped the $S$ to process the available stereoscopic cues.

\section{Experiment 2}

The attempt to determine the amount of time required for stereoscopic information processing by varying the interval between the stereo test and a blanking field of randomly distributed (in the threedimensional viewing space) dots resulted in a function exhibiting a marked discontinuity at about $50 \mathrm{msec}$ as Fig. 6 shows. This finding is in agreement with the estimate of stereoscopic perception time made by Julesz (1964). The results of Experiment 2 shown in Fig. 6 are plotted as a function of interval with all disparity and duration conditions pooled. At the moderate masking densities used in this experiment, only one of the three Ss was completely reduced to chance levels of performance at the shortest intervals. The average performance level was 59 per cent when a 1 -msec interval between the stereoscopic test and the mask was employed. As the separation increases, there is only a slight increase in performance until the interval of $50 \mathrm{msec}$ is reached. At that point, an abrupt increase to a 68 per cent performance level is observed. For intervals greater than $50 \mathrm{msec}$ there is again little improvement. The fact that there is sharp discontinuity at $50 \mathrm{msec}$ suggests that approximately this amount of time is necessary for the establishment of the perception of depth.

This finding is quite dissimilar to the function obtained for backward masking of two-dimensional stimuli. In that case, there is a continual increase after a 10-msec initial plateau (Uttal, 1973) confirming the suggestion by Sperling (1963) that only $10 \mathrm{msec}$ is required for the processing of simple two-dimensional stimuli.

The data have been further parsed out in Figs. 7 and 8 for the two exposure durations and the set of disparities that were used. Figure 7 shows the results for the 40-msec exposure duration. The curves have been plotted as a function of disparity with the interval between 


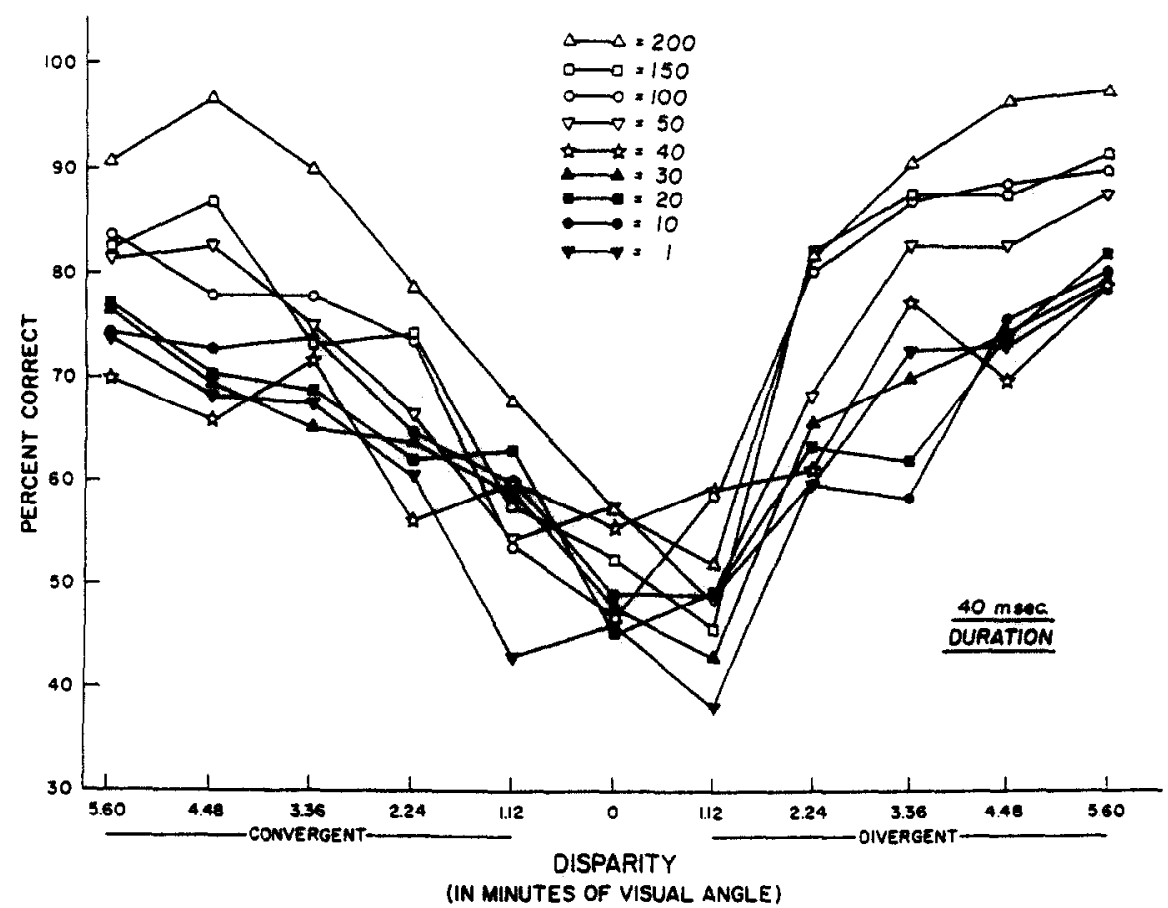

Fig. 7. The data for exposure durations of $40 \mathrm{msec}$ that were pooled in Fig. 6 are presented separately in this figure. The family of curves is parametric with the interval between the stimulus and the mask measured in msec.

the stimulus and blanking mask as a parameter. The decline in the performance with both decreasing disparity and decreasing interval is clearly evident. Figure 8 presents similar data for the 20 -msec viewing condition. In this case, the lower absolute levels of these curves relative to those of the 40 -msec viewing condi- tion further illustrate the decrease in performance associated with shortened viewing times. Figure 8 exhibits an asymmetry between the performance levels of the convergent and divergent disparity conditions. Convergent values less than 50 -msec display a shallower slope than the divergent values.

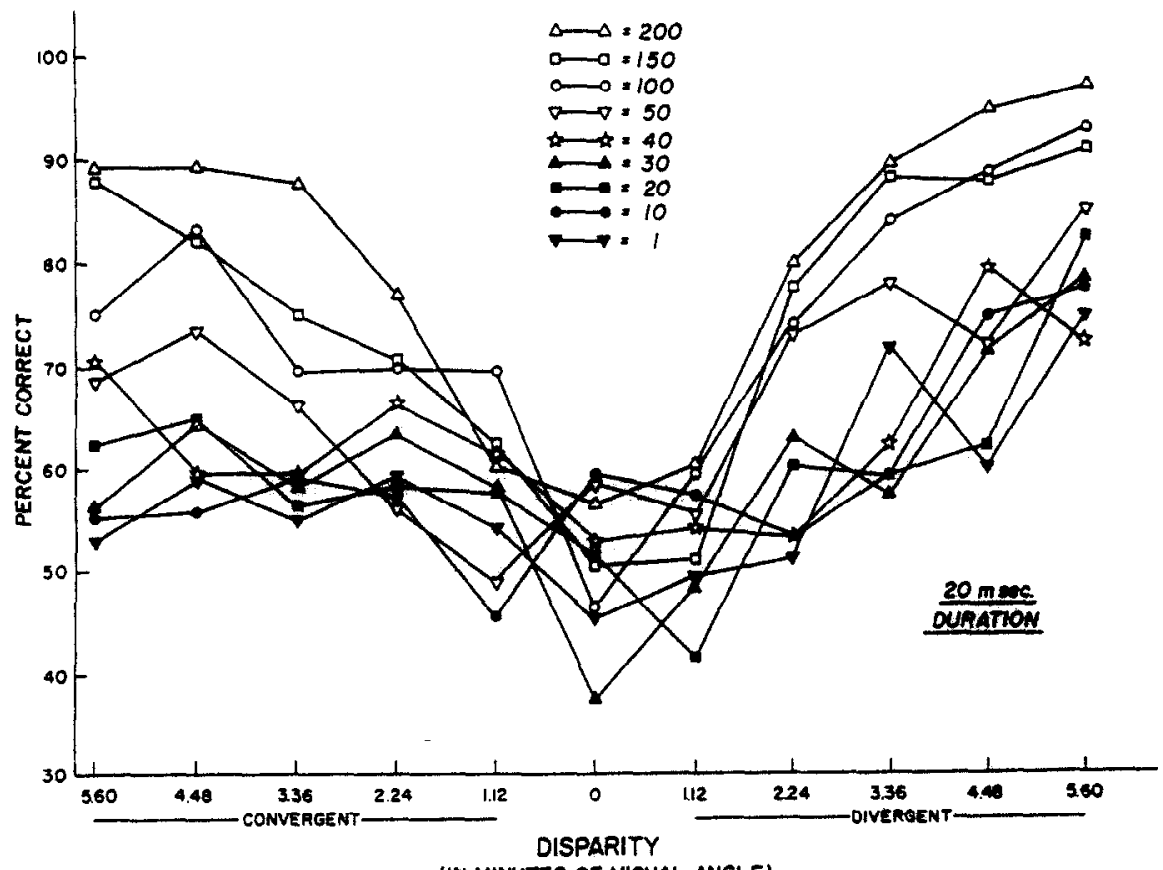

(IN MINUTES OF VISUAL ANGLE)

Fig. 8. The data for exposure durations of $20 \mathrm{msec}$ that were pooled in Fig. 6 are presented separately in this figure. Note the asymmetry in this case. The family of curves is parametric with the interval between the stimulus and the mask measured in msec. 


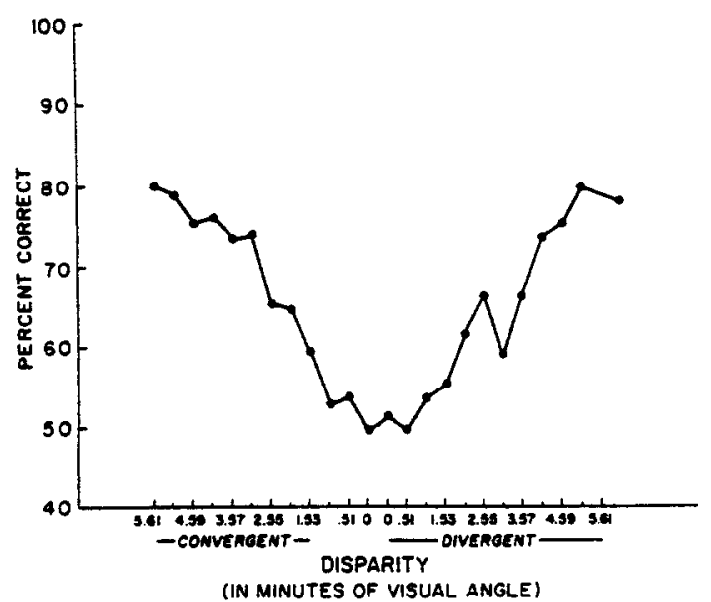

Fig. 9. The results of Experiment 3 for a severely reduced stimulus consisting only of two dots presented at the briefest possible durations. Disparity sensitivity remains high and continuously and symmetrically improves with increases in either convergent or divergent disparity. The minimum detectable disparity remains less than 1 ' of visual angle-even with this impoverished stimulus. Values for 0 disparity were collected twice and not pooled in this figure.

\section{Experiment 3}

The third experiment reduced all stimulus conditions to their minimums. However, $S$ s were still able to perform at a creditable level as shown in Fig. 9. Performance levels ranged from 80 per cent at the greatest disparities down to chance levels when the disparity was zero. Both crossed and uncrossed disparities seem to produce comparable results with the function being nearly symmetical around the zero disparity condition. The slight elevation of the curve for disparities of $30^{\prime \prime}$ of visual angle above that of the zero disparity condition suggests that there is an ability of the part of the Ss. in some instances, to discriminate stereoscopic depth on the basis of disparities of this order of magnitude. Although $30^{\prime \prime}$ of visual angle is a considerably higher value than that usually cited for bright and prolonged stimuli (thresholds of only a few seconds of visual angle are often reported and even then at the 75 per cent correct performance level) these values are comparable to those obtained with other dim, tachistoscopically exposed stimuli. Furthermore, the total freedom of the Julesz-type stimulus from any potential secondary monocular depth cue makes this estimate of the threshold of disparity sensitivity particularly compelling.

\section{DISCLSSION}

The findings obtained in the present study add little that is new to mathematical or physiological theories of how dichoptic views are fused in stereoscopic percepts. The works of Ogle (1950), Julesz (1971), and other researchers in the field have spoken:to the-many issues involved in theorizing about mathematical and neural models of stereoscopic perception. The work reported here, however, does provide a more complete description of some of the factors that influence stereoscopic depth perception. particularly with regard to temporal properties, disparity functions, and the effect of density in random dot stereopsis.
Let us consider the problem of the stereoscopic threshold first. Graham (1965) reviewed many experiments that must now be regarded as the classic investigations of stereoscopic thresholds. He cites the work of Howard (1919) who, using conventional two-stick measuring devices, determined the threshold ( 75 per cent correct level) to be of the order of $2 \cdot 0^{\prime \prime}$ of visual angle, a value very close to that obtained by Woodburne (1934). Graham goes on to remind us, however, that all of the studies used relatively long exposures and that when brief exposures were used by Langlands (1926). the thresholds were considerably elevated-up to about $20-40^{\prime \prime}$ of visual angle.

The results of the present experiment point to minimum detectable disparities that lie closer to $30^{\prime \prime}$. Furthermore, a 75 per cent proportion of correct responses is not obtained in the present study until much greater disparities are introduced. Nevertheless, the stimulus conditions of the Julesz-type random dot stereogram are so totally devoid of any possible secondary cue (such factors as angular separation of the sticks and stick width substantially affect threshold measurements in the Howard-Dohlman apparatus) that the values obtained in the present experiment may be considered to be especially useful estimates of the threshold of pure stereopsis.

It is also known that stereoscopic threshold is a function of luminance (Mueller and Lloyd, 1948) with thresholds varying from about $8 \mathrm{sec}$ to about $25 \mathrm{sec}$ as the stimulus illuminance varies from " $-4 \log \mathrm{mL}$ " to $" 2 \log \mathrm{mL}$. Since the present experiment used relatively dim stimuli, this function may also be contributing to the moderately high values for the obtained thresholds.

We have also shown that the effect on stereopsis of the number of dots in the display depends upon the exposure duration. Prolonged displays are only minimally affected by simple numerosity; the differential effect of dot numerosity disappears when more than ten dots are added to the display. Thus, at these longer durations, it appears that little statistical advantage would be obtained from increasing the stimulus dot density beyond relatively low values. For shorter exposure duration, however, the effect of dot density is somewhat enhanced and there are noticeable differences between the scores for the 30- and 50-dot displays. However, it should be noted that stereopsis is still powerfully compelling with even two dots for the shortest exposure durations and the smallest disparities if the convergence is tightly controlled.

Our findings suggest that there is only weak interaction of the neural mechanisms among brain mechanisms to enhance depth perception. Rather, whatever processes exist probably operate more or less independently to encode the depth of individual regions but do not substantially reinforce the overall response strength by collective interaction. Since adjoining stimulus areas may be at different real depths, this is an altogether adaptive response characteristic.

Finally, with regard to the contribution of perception time and/or viewing time in the establishment of stereoscopic depth in random dot stereograms, we have provided two different pieces of information. First, as exhibited in Experiment 1, a reduction in viewing time per se does produce a reduction in the percentage of correct depth discriminations. This 
occurs in spite of the fact that the persistence of the visual image-the temporal spread of the impulsive stimulus-is certainly much longer than the stimulus duration at the shorter exposure durations. Since the function depicting the effect of duration is more or less continuous to durations too brief to allow vergence adjustments. it seems likely that the duration of the stimulus is a significant factor itself in the perception of depth. independent of the influence of further artifacts such as oculomotor responses.

Second, the minimum period required for the perception of stereoscopic depth. as reflected in the discontinuity observed at $50 \mathrm{msec}$ in Experiment 2, is somewhat longer than some of the estimates of the period of interaction between sequential two-dimensional stimuli. Measures of the period of sequential interaction have been made using techniques very similar to the procedures of the present study (Uttal. 1971). There is very little residual interaction between twodimensional stimuli when the intervals between sequential stimuli were greater than $40 \mathrm{msec}$. Thus the process occurring in the $50 \mathrm{msec}$ required for stereopsis could benefit from prolonged stimulus durations that would provide the responsible neural mechanisms with a continuing effective stimulus not provided by simple persistence.

It should also be noted that stereoscopic stimuli can have prolonged effects following the cessation of the stimulus under other conditions. A number of recent studies (Mitchell and Baker, 1973; Blakemore and Julesz, 1971) have shown persistent after-effects of stereoscopic inspection patterns that produce gross errors (up to $100^{\prime \prime}$ of visual angle in compensatory directions) for up to $2 \mathrm{~min}$. These observations suggest that whatever mechanisms account for stereopsis, they are dependent upon the time course of previous events in an especially sensitive manner.

Since the nature of the temporal functions for stereopsis and two-dimensional vision are so dissimilar. we may conclude that two-dimensional vision and three-dimensional vision may have substantially different time constants and may thus reflect the action of quite different neural mechanisms. This notion of a group of relatively independent visual skills keeps recurring in much of the work carried out in our laboratory.

\section{REFERENCES}

Blakemore C. and Julesz B. (1971) Stereoscopic depth aftereffect produced without monocular cues. Science 171. 286-288.

Dove H. W. (1841) Uber stereoskipie. Annin Phys. 110 (Series 2), 494-498.

Graham C. H. (1965) Vision and Visual Perception. Wiley, New York.

Howard H. J. (1919) A test for the judgment of distance. Am. J. Optom. 2, 656-675.

Julesz B. (1964) Binocular depth perception with familiarity cues. Science 145. 356-362.

Julesz B. (1971) Foundations of Cyclopean Perception. University of Chicago Press Chicago.

Langlands H. M. S. (1926) Experiments in binocular vision. Trans. opt. Soc. 28, 45-82.

Marlow L. H. (1969) Orientation of contours and binocular depth perception. Ph.D. Dissertation, Brown University.

Mitchell D. E. and Baker A. G. (1973) Stereoscopic aftereffects: evidence for disparity-specific neurones in the human visual system. Vision Res. 13, 2273-2288.

Mueller C. B. and Lloyd V. V. (1948) Stereoscopic acuity for various levels of illuminance. Proc. natn. Acad. Sci. U.S.A. 34, 223-227.

Ogle K. N. (1950) Researches in Binocular Vision. Saunders, Philadelphia.

Papert S. (1964) Stereoscopic synthesis as a technique for localizing visual mechanisms. M.I.T. Quarterly Progress Report 73, 239-243.

Ross J. (1974) Stereopsis by binocular delay. Nature. Lond. $248,363-364$

Ross J. and Hogben J. H. (1974) Short-term memory in stereopsis. Vision Res. 14, 1195-1201.

Sperling G. (1963) A model for visual memory tasks. Hum. Factors 5, 19-31.

Uttal W. R. (1971) The effect of interval and number on masking with dot bursts. Percept. Psychophys. 9, 469-473.

Uttal W. R. (1973) Chromatic and intensive effects in dotpattern masking: Evidence for different time constants in color vision. J. opt. Soc. Am. 63, 1490-1494.

Woodburne L. S. (1934) The effect of a constant visual angle upon the binocular discrimination of depth differences. Am. J. Psychol. 46, 273-286. 